\title{
Solvation of anthraquinone and Tempo redox-active species in acetonitrile using a polarizable force field
}

\author{
Roxanne Berthin, ${ }^{1,2}$ Alessandra Serva, ${ }^{1,2, \text { a) }}$ Kyle G. Reeves, ${ }^{1,2}$ Esther Heid, ${ }^{3}$ Christian Schröder, ${ }^{3}$ and Mathieu \\ Salanne $1,2,4$, b) \\ 1) Sorbonne Université, CNRS, Physico-chimie des Électrolytes et Nanosystèmes Interfaciaux, PHENIX, \\ F-75005 Paris \\ ${ }^{2)}$ Réseau sur le Stockage Electrochimique de l'Energie (RS2E), FR CNRS 3459, 80039 Amiens Cedex, \\ France \\ ${ }^{3)}$ University of Vienna, Faculty of Chemistry, Department of Computational Biological Chemistry, \\ Währingerstraße 19, A-1090 Vienna, Austria \\ 4) Institut Universitaire de France (IUF), 75231 Paris Cedex 05, France
}

\begin{abstract}
Redox active molecules are of interest in many fields such as medicine, catalysis or energy storage. In particular, in supercapacitor applications, they can be grafted to ionic liquids to form so-called biredox ionic liquids. To completely understand the structural and transport properties of such systems, an insight at the molecular scale is often required but few force fields are developed ad hoc for these molecules. Moreover, they do not include polarization effects, which can lead to inaccurate solvation and dynamical properties. In this work, we developed polarizable force fields for redox-active species anthraquinone (AQ) and 2,2,6,6-tetra-methylpiperidinyl-1-oxyl (TEMPO) in their oxidized and reduced states, as well as for acetonitrile. We validate structural properties of $\mathrm{AQ}, \mathrm{AQ}^{\bullet-}, \mathrm{AQ}^{2-}, \mathrm{TEMPO}$ and $\mathrm{TEMPO}^{+}$in acetonitrile against density functional theory-based molecular dynamics simulations and we study the solvation of these redox molecules in acetonitrile. This work is a first step toward the characterization of the role played by $\mathrm{AQ}$ and TEMPO in electrochemical and catalytic devices.
\end{abstract}

\section{INTRODUCTION}

Redox-active molecules, such as anthraquinone (AQ) and 2,2,6,6-tetra-methylpiperidinyl-1-oxyl (TEMPO), have recently emerged as very promising species in many fields, ranging from medical applications, ${ }^{1}$ catalysis $^{2,3}$ to energy storage devices, such as redoxflow batteries ${ }^{4-6}$ and supercapacitors ${ }^{7}$. For example, in supercapacitor applications, redox-active molecules can be grafted to ionic liquids, often employed as electrolytes in such devices, in order to form so-called biredox ionic liquids (BILs). ${ }^{8}$ Through redox processes (the cation can be oxidized and the anion can be reduced), BILs can exchange electrons with the electrode, leading to a significant increase of the capacitance of the system..$^{9,10}$

In a previous work, we have investigated the electrochemical properties of these redox-active molecules dissolved in acetonitrile by means of density functional theory (DFT)-based Molecular Dynamics (MD) simulations, where we employed a two Gaussian state model in order to accurately calculate the redox reaction free energies. ${ }^{11}$ However, in order to obtain a better understanding of some of their properties (e.g. transport coefficients such as the self-diffusion coefficients), as well as to characterize larger and more complex systems, for example, to explicitly model their

\footnotetext{
a) Electronic mail: alessandra.serva@sorbonne-universite.fr

b) Electronic mail: mathieu.salanne@sorbonne-universite.fr
}

adsorption on electrodes in supercapacitor systems, classical MD simulations are required. Several force fields, such as OPLS and AMBER, already exist in the literature to model organic molecules and some others have been developed ad hoc for the TEMPO redox species. ${ }^{12}$ Nevertheless, it is well known that polarization effects play a key role in the description of transport properties of ionic liquids. Taking into account polarization for the redox species can thus be relevant when they are grafted to ionic liquids. Their organization in the vicinity of a surface under an applied electric field may also be affected.

In this work, using a well-established fitting procedure based on DFT reference calculations, ${ }^{13,14}$ we develop a polarizable force field for $\mathrm{AQ}$ and TEMPO species, in both their oxidized and reduced states. The advantage of this method is that no experimental information is used during the development, which generally improves the transferability of the parameters obtained. A schematic representation of the molecules is provided in Figure 1. Since we start by investigating their properties in acetonitrile, a new polarizable force field for the solvent is also derived. This guarantees that the same level of description/accuracy is used for both the redox moieties and the solvent.

After validating the new force field, we provide a general overview of the solvation properties of the investigated systems. 

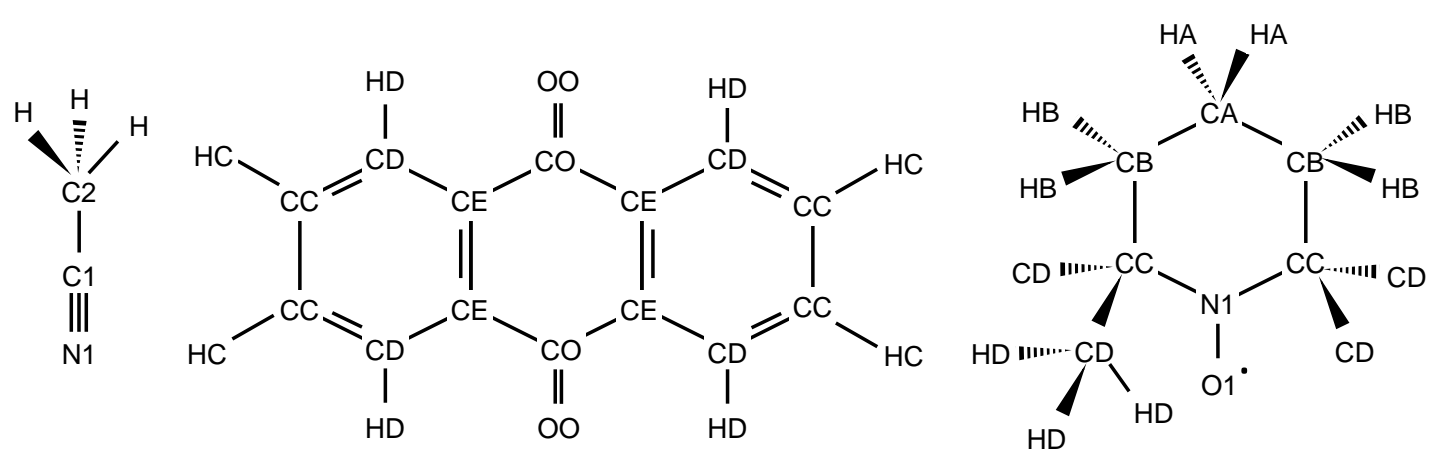

FIG. 1. Molecular structure of acetonitrile (ACN), anthraquinone (AQ) and 2,2,6,6-tetra-methylpiperidinyl-1-oxyl (TEMPO), together with the atom labels used in this work.

\section{METHODS}

\section{A. Force-field model}

The intramolecular potential is described using Eq. 1:

$$
\begin{array}{r}
\left.V_{\text {intra }}=\sum_{\text {bonds }} k_{0, r}\left(\left|r_{i j}\right|-r_{0}\right)^{2}+\sum_{\text {angles }} k_{0, \theta}\left(\left|\theta_{i j k}\right|-\theta_{0}\right) \mathbb{1}^{2}\right) \\
+\sum_{\text {dihedrals }} 0.5\left[v_{1}\left(1+\cos \left(\phi_{i j k l}\right)\right)+v_{2}\left(1-\cos \left(2 \phi_{i j k l}\right)\right)\right. \\
\left.+v_{3}\left(1+\cos \left(3 \phi_{i j k l}\right)\right)+v_{4}\left(1-\cos \left(4 \phi_{i j k l}\right)\right)\right]
\end{array}
$$

A scaling factor of 0.5 is used for the 1-4 electrostatic and Van der Waals interactions, similarly to the OPLS force field.

Intermolecular interactions are described following Eq. 2. The first term corresponds to the charge-charge electrostatic interaction, where $q^{i}$ and $q^{j}$ are the partial charges on atoms $i$ and $j$. The two following terms represent a Fumi-Tosi potential ${ }^{15,16}$; they consist of a repulsion part and a dispersion part,

$$
\begin{aligned}
V_{\text {inter }}= & \sum_{i<j}\left(\frac{q^{i} q^{j}}{r^{i j}}+A^{i j} \exp \left(-B^{i j} r^{i j}\right)\right. \\
& \left.-f_{6}^{i j} \frac{C_{6}^{i j}}{\left(r^{i j}\right)^{6}}-f_{8}^{i j} \frac{C_{8}^{i j}}{\left(r^{i j}\right)^{8}}\right)+V_{\text {pol }}
\end{aligned}
$$

with $C_{6}^{i j}$ and $C_{8}^{i j}$ the dipole-dipole and the dipolequadrupole dispersion coefficients, respectively. Combinations rules used for different atom types can be found in the SI. Tang-Toennies dispersion damping functions ${ }^{17} f_{n}^{i j}$ (Eq. 3) stand for the short-range penetration correction to the asymptotic multipole expansion of dispersion, where the $B_{n}^{i j}$ parameter sets the range of the damping effect.

$$
f_{n}^{i j}\left(r^{i j}\right)=1-\exp \left(-B_{n}^{i j} r^{i j}\right) \sum_{k=0}^{n} \frac{\left(B_{n}^{i j} r^{i j}\right)^{k}}{k !}
$$

The last term of Eq. 2 refers to the polarization part of the potential (Eq. 4), where $\alpha_{i}$ is the polarizability of atom $i, \mu_{i}$ are the induced dipoles and $\mathbf{T}_{\alpha}^{(1)}\left(r^{i j}\right)=$ $-r_{\alpha} / r^{3}, \mathbf{T}_{\alpha \beta}^{(2)}\left(r^{i j}\right)=\left(3 r_{\alpha} r_{\beta}-r^{2} \delta_{\alpha \beta}\right) / r^{5}$ are the chargedipole and dipole-dipole interaction tensors, respectively.

$$
\begin{array}{r}
V_{\text {pol }}=\sum_{i j}\left[\left(q^{i} \mu_{\alpha}^{j} g^{i j}\left(r^{i j}\right)-\left(q^{i} \mu_{\alpha}^{i} g^{j i}\left(r^{i j}\right)\right) \mathbf{T}_{\alpha}^{(1)}\left(r^{i j}\right)\right.\right. \\
\left.-\mu_{\alpha}^{j} \mu_{\beta}^{j} \mathbf{T}_{\alpha \beta}^{(2)}\right]+\sum_{i}\left(\frac{1}{2 \alpha^{i}}\left|\mu^{i}\right|^{2}\right)(4)
\end{array}
$$

Here also, Tang-Toennies functions $g_{n}^{i j}\left(r^{i j}\right)$ (Eq. 5) are included to account for the short-range damping effects, with $c^{i j}$ the strength of the ion response to the short-range effects. All atoms pairs $i j$ have the same damping functions parameters, $n=4, b_{4}^{i j}=2.0 \mathrm{a.u.}^{-1}$ and $c_{4}^{i j}=1.0$, in order to reduce the number of fitting parameters.

$$
g_{n}^{i j}\left(r^{i j}\right)=1-c_{n}^{i j} \exp \left(-b_{n}^{i j} r^{i j}\right) \sum_{k=0}^{n} \frac{\left(b_{n}^{i j} r^{i j}\right)^{k}}{k !}
$$

Unlike the partial charges, which are fixed parameters, the induced dipoles are additional degrees of freedom which are calculated self-consistently at each time step. In practice, this is made by minimization of $V_{p o l}$ through a conjugate gradient procedure ${ }^{18,19}$ albeit alternative approaches involving constrained molecular dynamics techniques have recently been proposed ${ }^{20}$.

\section{B. Force-field fitting procedure}

Six systems have been considered during the fitting procedure, pure $\mathrm{ACN}, \mathrm{AQ}$ in $\mathrm{ACN}, \mathrm{AQ}^{--}$in $\mathrm{ACN}$, 


\begin{tabular}{|c|ccccc|c|c|}
\hline System & \multicolumn{4}{|c|}{ Pure ACN } & AQ/ACN & TEMPO/ACN \\
\hline N(ACN) & 216 & 500 & 800 & 1400 & 2000 & 95 & 96 \\
Box length $(\AA)$ & 26.10 & 35.13 & 41.08 & 49.51 & 55.76 & 20.55 & 20.59 \\
Intermolecular cutoff $(\AA)$ & 13.23 & 15.00 & 15.00 & 15.00 & 15.00 & 10.05 & 10.05 \\
Simulation time $(\mathrm{ns})$ & 10 & 10 & 10 & 20 & 20 & 50 & 50 \\
\hline
\end{tabular}

TABLE I. MD simulations details. For AQ and TEMPO simulation parameters are the same for the oxidized and reduced state.

$\mathrm{AQ}^{2-}$ in $\mathrm{ACN}, \mathrm{TEMPO}^{\bullet}$ in $\mathrm{ACN}$ and $\mathrm{TEMPO}^{+}$in ACN. Note that for all species, intramolecular equilibrium distances and angles $\left(r_{0}\right.$ and $\left.\theta_{0}\right)$ are fixed to the average values obtained from our previous DFT-MD simulations. ${ }^{11}$

- For acetonitrile and non-radical species, i.e AQ and $\mathrm{TEMPO}^{+}$, ten representative configurations were taken. On each configuration, forces and induced dipoles were computed at the DFT level. Details of these calculations are provided in the Supplementary Information. All force-field parameters have been fitted, with the exception of the $r_{0}$ and $\theta_{0}$ values.

- For $\mathrm{AQ}^{\bullet-}$ and $\mathrm{AQ}^{2-}$, we kept all the fitted parameters of AQ (i.e. force constants for bonds and angles, dihedral parameters and Tosi-Fumi parameters). Only the atomic charges were changed: new atomic charges for $\mathrm{AQ}^{\bullet-}$ and $\mathrm{AQ}^{2-}$ were obtained by subtracting 0.0625 and 0.125 from the atomic charges of the carbons and the oxygens of AQ, respectively. Atomic charges for hydrogen atoms were not modified.

- For TEMPO•, we fitted force constants for bonds, angles and dihedral parameters and we kept the Tosi-Fumi parameters of $\mathrm{TEMPO}^{+}$. The atomic charges were obtained by adding 0.1 to the CA, $\mathrm{CB}$ and $\mathrm{CC}$ carbons, 0.05 to the $\mathrm{CD}$ carbons and 0.15 to $\mathrm{N}$ and $\mathrm{O}$ atoms (see Figure 1 for the atom labels).

Atomic polarizabilities, $\alpha_{i}$, were left as free parameters in the case of acetonitrile. For the redox species, they were calculated using a specific procedure developed by Heid et al.21,22. For each redox molecule, single-point calculations were run with an applied electric field of 0.0008 a.u. in the positive and negative $x$, $y$ and $z$ directions. These six single-point calculations were performed at the DFT level using the Gaussian09 package, ${ }^{23}$ with the UB3LYP + D3 functional and def2TZVPPD basis set. Using GDMA, ${ }^{24,25}$ atomic dipoles were calculated, allowing us to finally obtain atomic polarizabilities. Since the DFT calculations were performed in the gas state, we applied a scaling factor of 0.7 to the computed atomic polarizabilities for use in the liquid state. ${ }^{26}$
Using an iterative procedure, the potential parameters are fitted by minimizing the deviation $\chi^{2}(\mathbf{A})$ (Eq. 6) of $\mathbf{A}_{i}^{D F T}$ (which is either the set of forces or dipoles) obtained from the DFT calculations.

$$
\chi^{2}(\mathbf{A})=\frac{\sum_{i}^{N}\left|\mathbf{A}_{i}^{D F T}-\mathbf{A}_{i}^{F F}\right|^{2}}{\sum_{i}^{N}\left|\mathbf{A}_{i}^{D F T}\right|^{2}}
$$

Although the prediction of the density of the systems should be improved by the use of dispersion corrections in the DFT calculation ${ }^{27}$, the agreement with experiments is not perfect for the latter. To overcome this shortcoming, at the end of the fitting procedure, the $C_{6}^{i j}$ and $C_{8}^{i j}$ Fumi-Tosi parameters were empirically scaled by a factor 1.08 in order to recover the experimental density of acetonitrile.

All force-field parameters for both acetonitrile and the redox-active molecules are summarized in Tables S1 to S6 in the SI.

Classical MD simulations were performed using the MetalWalls code. ${ }^{28}$ Details of the simulations are reported in Table I. In the case of the redox systems, simulation cells were made of one redox molecule (either $\mathrm{AQ}, \mathrm{AQ}^{\bullet-}, \mathrm{AQ}^{2-}, \mathrm{TEMPO}^{\bullet}$ or $\mathrm{TEMPO}^{+}$) dissolved in acetonitrile. Cubic cells were built using the PACKMOL package. ${ }^{29}$ A first NPT run at $298 \mathrm{~K}$ and $1 \mathrm{~atm}$ was performed to reach the experimental density of acetonitrile. Then, the simulations were carried out in the NVT ensemble (at a fixed temperature of $298 \mathrm{~K}$ ), after $1 \mathrm{~ns}$ of equilibration. A timestep of $1 \mathrm{fs}$ was used and the trajectory was saved every 500 fs. The equations of motion were integrated using the velocity-Verlet algorithm and the temperature was kept constant using a chain Nosé-Hoover thermostat. ${ }^{30,31}$ The Ewald summation method was used for electrostatic interactions. Cutoff values for van der Waals interactions are listed in Table I.

Radial, dihedral and spatial distribution functions as well as structure factors were calculated using the TRAVIS software. ${ }^{32}$ All other analyses were performed with in-house written codes. 


\section{RESULTS AND DISCUSSION}

\section{A. Pure acetonitrile}

In order to analyze the liquid structure, we calculated radial distribution functions, RDFs, of a selected subset of atoms from the MD trajectory (see Figure 2, top and middle panels, and Supplementary Figure S1). The strongest interaction is between the nitrogen atom and the methyl group (N1-C2), with a first sharp peak in the RDF due to the head-tail attractive electrostatic interactions.

In general, as visibile in Figure 2 and Supplementary Figure S1, the RDFs obtained using our new force field compare well with those obtained in a previous DFT-MD simulation ${ }^{11}$. There is, however, a small difference for the N1-H RDF (Figure 2, middle panel) that indicates small differences in the local liquid structure. The DFT-MD-calculated N1-H RDF features two broad peaks, while a first intense peak followed by a less intense peak are present in the classical one. These two peaks can respectively be assigned to the case where two $\mathrm{H}$ atoms belonging to the same methyl group are coordinated with the N1 atom of another molecule in a bidentate mode for the former, and to a monodentate coordination involving a single $\mathrm{H}-\mathrm{N}$ contact for the latter. The population of the two coordination modes slightly differs between the two methods. We furthermore note that the obtained RDFs are in agreement with the ones obtained from previous non-polarizable six-site models by Grabuleda et $a l .{ }^{33}$ and Nikitin et $a l .{ }^{34}$, and the three-site models of References 35-38.

The integration of the C1-C1 RDF of Figure S1 up to the first minimum (around $6 \AA$ ) is a measure of the number of ACN molecules in the first solvation shell of a reference acetonitrile molecule. We found approximately 12 molecules, which is in agreement with $\mathrm{X}$ ray diffraction ${ }^{40}$ as well as neutron scattering ${ }^{41}$ experiments. Moreover, we observe a good agreement between the X-ray diffractogram calculated using our force field and the experimental one ${ }^{39}$ (see Figure 2, bottom panel).

The infrared spectrum of liquid acetonitrile was also calculated from the imaginary part of the total dielectric function, following the procedure of Caillol et al. ${ }^{43,44}$. The spectrum is shown in Figure 3, and compared to the experimental result. ${ }^{42}$ For convenience, we convert the imaginary part of the total dielectric function into absorption coefficients. In the experimental spectrum, one can identify seven bands. The comparison between the experimental and simulated frequencies are reported in Table II. Our force field is able to reproduce well the acetonitrile vibrational modes with deviations between 1 and $8 \%$
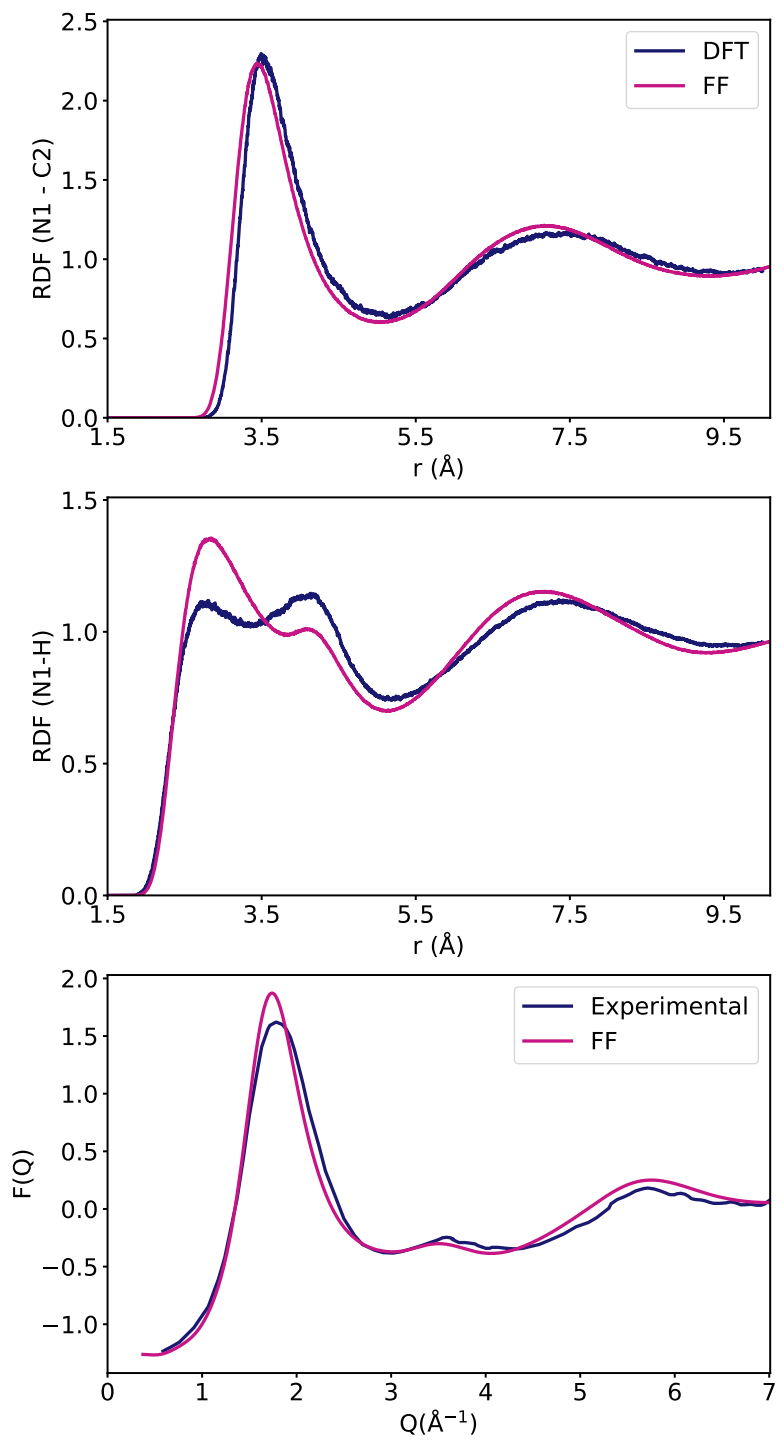

FIG. 2. Comparison between the intermolecular N1-C2 (top) and N1-H (middle) RDFs, calculated by DFT-MD simulation ${ }^{11}$ and those calculated using the fitted force field (FF). Bottom panel: comparison between the X-ray structure factor as obtained by experiment and calculated using the $\mathrm{FF}^{39}$

\begin{tabular}{lccc} 
& Exp. & FF & Error (\%) \\
\hline Sym. stretching & 3006 & 2990 & 5.32 \\
Asym. stretching & 2946 & 2889 & 1.94 \\
CN stretching & 2259 & 2232 & 1.20 \\
$\mathrm{CH}_{3}$ asym. deformation & 1448 & 1360 & 6.08 \\
$\mathrm{CH}_{3}$ sym. deformation & 1384 & 1280 & 7.51 \\
$\mathrm{CH}_{3}$ rocking & 1041 & 957 & 8.07 \\
C-C stretching & 920 & 853 & 7.28 \\
\hline
\end{tabular}

TABLE II. Characteristic frequencies $\left(\mathrm{cm}^{-1}\right)$ of the imaginary part of the dielectric function (see Figure 3) obtained from our force field, compared to experiments. 


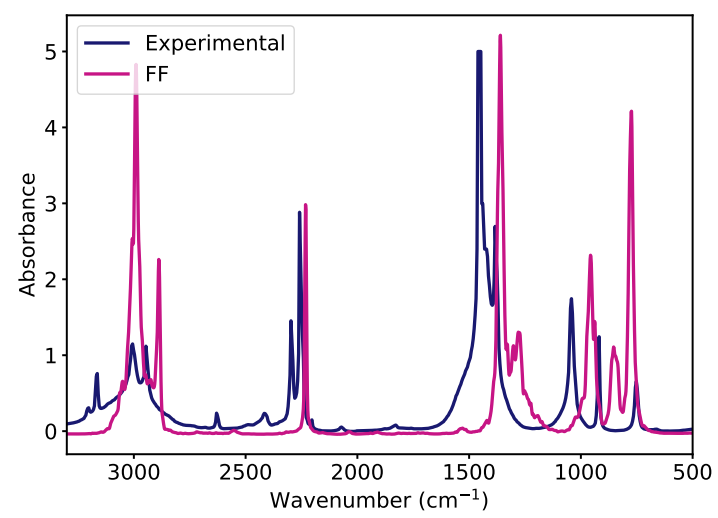

FIG. 3. Imaginary part of the dielectric function of liquid acetonitrile, calculated with the fitted force field, compared to experiments. ${ }^{42}$

from the experimental values. The largest deviations are found for $\mathrm{CH}_{3}$ symmetrical and asymmetrical deformation, $\mathrm{CH}_{3}$ rock and C-C stretch, but it should be kept in mind that $\mathrm{CH}_{3}$ deformation may include quantum effects that we cannot reproduce in our classical simulations.

Other properties of liquid acetonitrile (namely heat of vaporization, dielectric constant, diffusion coefficient and viscosity) were also computed. They are summarized in Table S7 in the SI and compared to the respective values obtained with force fields from the literature $^{33,34,45,46}$ as well as to experimental data ${ }^{47-58}$; a good agreement is observed for all properties.

\section{B. Redox-active species}

\section{Anthraquinone}

In order to assess the validity of the parameters for redox-active species, we have first computed a series of RDFs between anthraquinone and acetonitrile for all redox states, namely $\mathrm{AQ}, \mathrm{AQ}^{\bullet-}$ and $\mathrm{AQ}^{2-}$. The results are shown in Figure 4 and Supplementary Figure S3, together with those obtained from DFT-MD simulations. In general, a good agreement is observed between the DFT and classical MD simulations, showing the ability of our force field to correctly describe the structural organization of acetonitrile around AQ at the various oxidation states.

In particular, the strongest interaction of the redox species and the acetonitrile occurs via the AQ-oxygen atom and the methyl group. The OO-C2 $\mathrm{RDF}$ is shown in Figure 5a for the three redox states (namely $\mathrm{AQ}, \mathrm{AQ}^{\bullet-}$ and $\mathrm{AQ}^{2-}$ ). We see that the more negatively charged the molecule is, the more intense is the RDF first peak while its position remains the same,
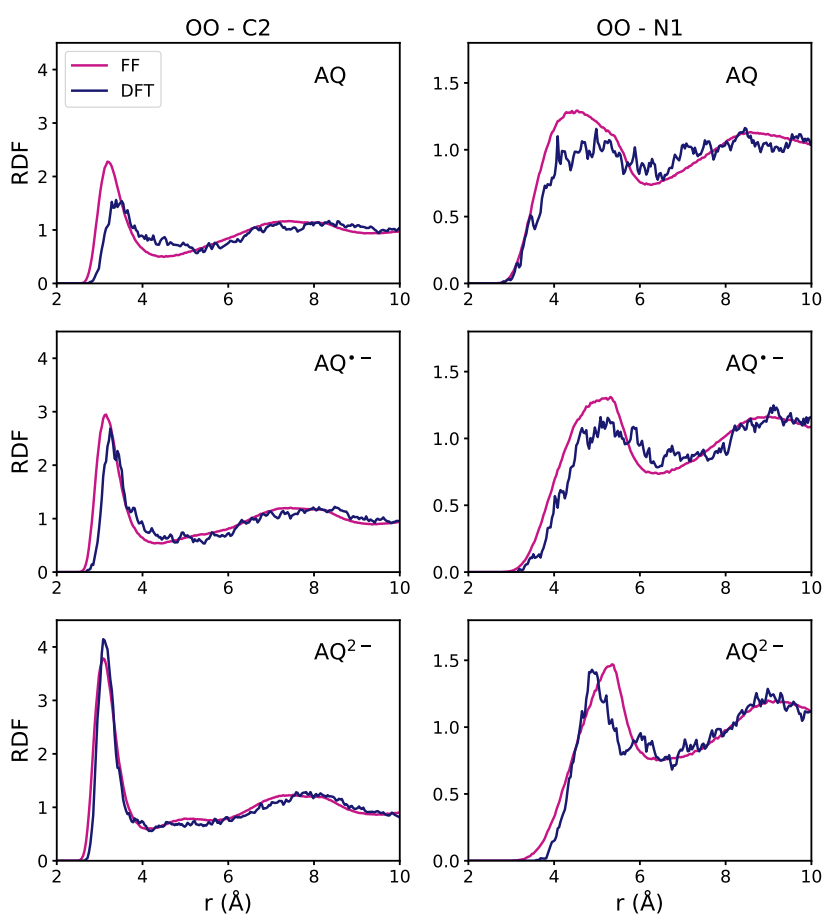

FIG. 4. Intermolecular anthraquinone-acetonitrile RDFs calculated from DFT-MD simulations (blue) and using our force field (magenta): OO-C2 (left), OO-N1 (right) for $\mathrm{AQ}$ (top), $\mathrm{AQ}^{\bullet-}$ (middle) and $\mathrm{AQ}^{2-}$ (bottom).

suggesting that the acetonitrile first solvation shell around the carbonyl group becomes more crowded. The increase in the number of ACN molecules is accompanied by a modification of their orientation as shown in Figure 5b, where the dihedral distribution function (DDF) between the carbonyl bond and the acetonitrile molecules belonging to the first solvation shell is reported. While there is no preferential orientation in the case of $\mathrm{AQ}^{\bullet-}$, acetonitrile molecules are well oriented in the case of $\mathrm{AQ}$ and $\mathrm{AQ}^{2-}$. In particular, for $\mathrm{AQ}^{2-}$ the prefered dihedral is about $\pm 180^{\circ}$, meaning that the acetonitrile molecules are facing the carbonyl bond of $\mathrm{AQ}^{2-}$, while a distribution centered at $0^{\circ}$ is observed in the case of AQ. The different behavior found for $\mathrm{AQ}$ and $\mathrm{AQ}^{2-}$ is visualized in Figure 5c, which depicts the spatial distribution functions, i.e. the 3-dimensional arrangement of acetonitrile around the redox molecules. For AQ, the methyl group of ACN (orange surface in Figure 5c) approaches only the area around the carbonyl bond, producing an empty space below and above the plane, while in the case of $\mathrm{AQ}^{2-}$ it is found all around the molecule. As a consequence, the nitrogen (blue surface in Figure 5c) is located at greater distance than the methyl group for $\mathrm{AQ}^{2-}$, whereas it fills the empty space for AQ. This observation is in agreement with the RDFs in Figure 4. A shift in the position of the first peak between the OO-N1 and the OO-C2 RDFs is found for $\mathrm{AQ}^{2-}$, while 
a.

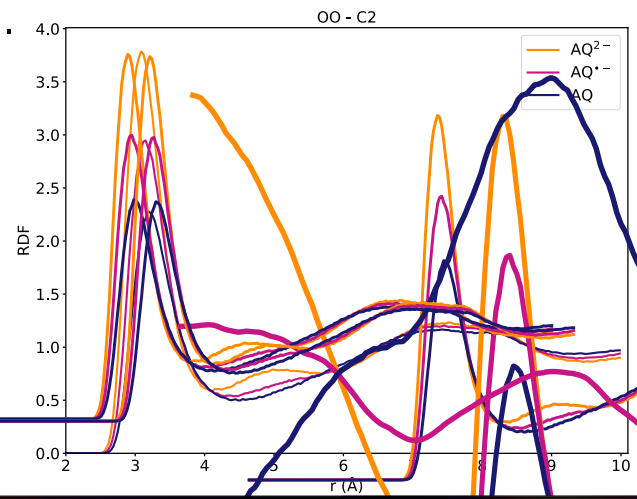

C.

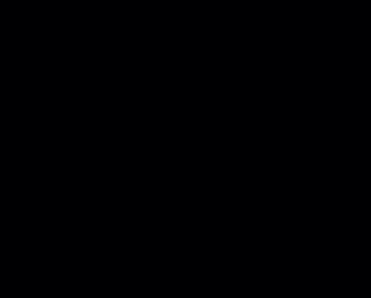

$\mathrm{AQ}$
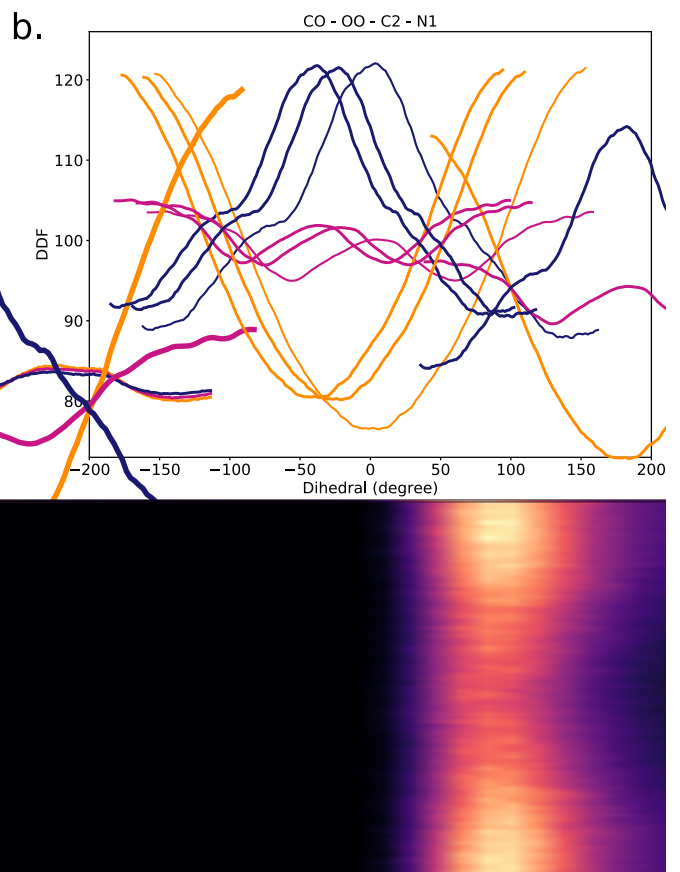

FIG. 5. a. Radial distribution function (RDF) between the oxygen of anthraquinone and the methyl carbon of acetonitrile for AQ (blue), $\mathrm{AQ}^{\bullet-}$ (magenta) and $\mathrm{AQ}^{2-}$ (orange). b. CO-OO-C2-N1 dihedral distribution function (DDF) between anthraquinone and acetonitrile for $\mathrm{AQ}, \mathrm{AQ}^{\bullet-}$ and $\mathrm{AQ}^{2-}$. The DDFs were computed considering only $\mathrm{ACN}$ molecules for which the distance between $\mathrm{OO}$ and $\mathrm{C} 2$ is below $4.0 \AA$. c. Spatial distribution functions (SDFs) of the nitrogen (N1, blue) and the methyl carbon (C2, orange) of acetonitrile around $\mathrm{AQ}$ (left) and $\mathrm{AQ}^{2-}$ (right). We use an isovalue of $30 \mathrm{~nm}^{-3}$ for $\mathrm{C} 2 \mathrm{and}^{25} \mathrm{~nm}{ }^{-3}$ for N1.

for AQ these two peaks have almost the same position.

\section{TEMPO}

In order to validate the force-field parameters of TEMPO-redox species, we first examine the structural organization of acetonitrile around them (Figure 6 and Supplementary Figure S4), analogously to AQ. We note that the NO moiety in TEMPO complicates the forcefield development procedure compared to $\mathrm{AQ}$, requiring a careful optimization of out-of-plane bending and torsion. The resulting force-field produces a good agreement between DFT and classical MD simulations.

In the following, we analyze the interaction between the NO moiety of TEMPO and acetonitrile in detail. The total number of ACN molecules in the first solvation shell (calculated with respect to the ACN center of mass, O1-OCM) is almost the same for TEMPO and $\mathrm{TEMPO}^{+}$(3.1 and 3.3, respectively). However, the distribution and orientation of acetonitrile around $\mathrm{TEMPO}^{\bullet}$ and $\mathrm{TEMPO}^{+}$differs significantly between them, visible in the RDFs for TEMPO-O and either the methyl group or the nitrogen atom of acetonitrile. For TEMPO ${ }^{\bullet}$, the NO oxygen is preferably coordinated to the ACN methyl group, visible from the large peak in the O1-C2 RDF (with a coordination number of
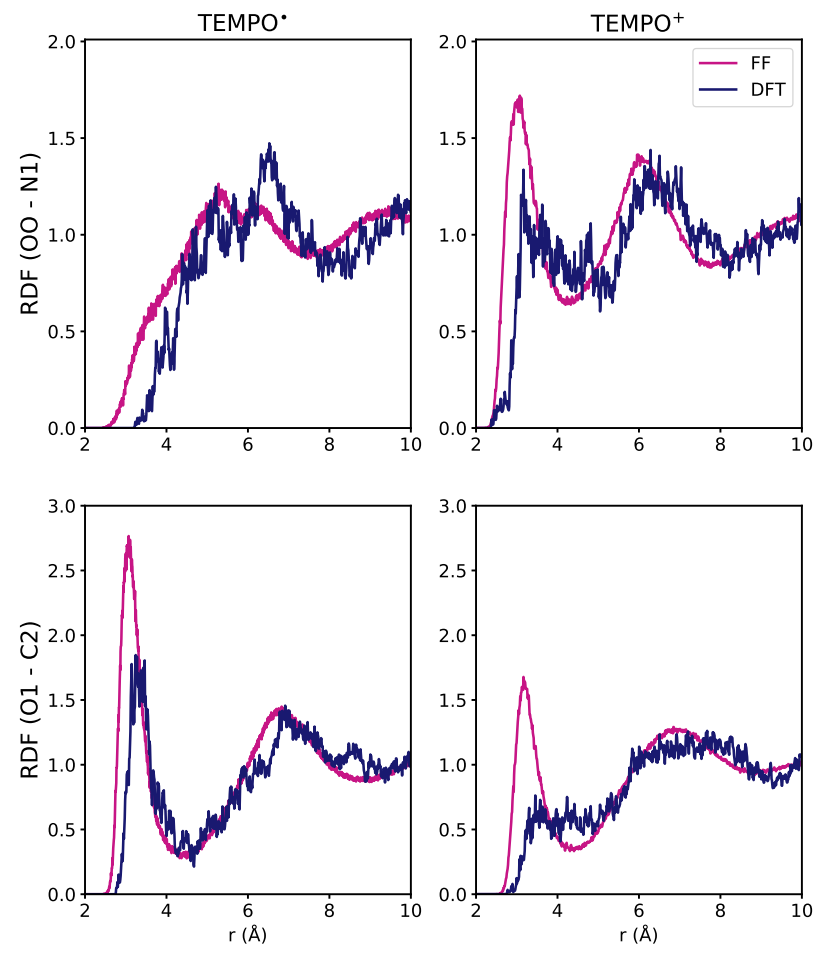

FIG. 6. Intermolecular TEMPO-acetonitrile RDFs calculated from DFT-MD simulations (blue) and using our force field (magenta): O1-N1 (top) and O1-C2 (bottom) for $\mathrm{TEMPO}^{\bullet}$ (first column) and $\mathrm{TEMPO}^{+}$(second column). 

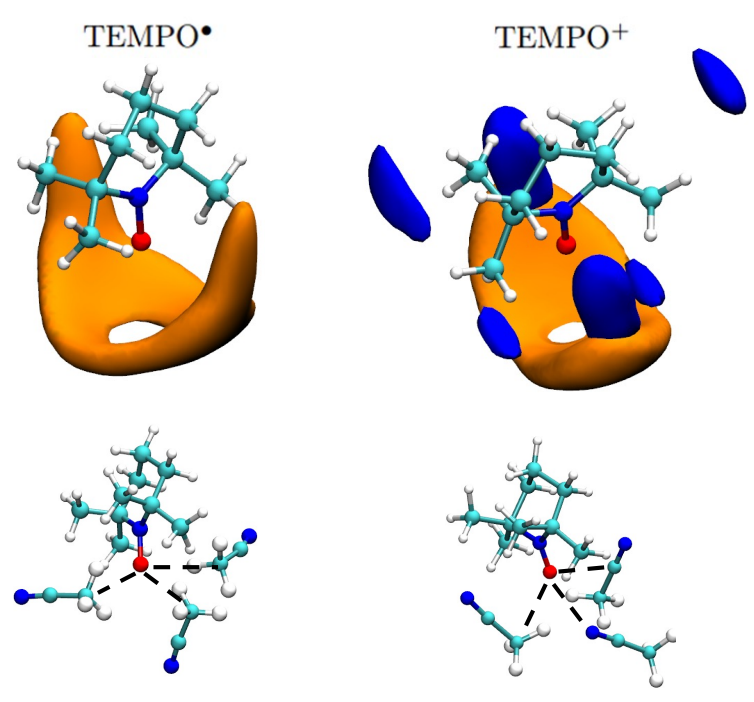

FIG. 7. Top panel: spatial distribution functions (SDFs) of the nitrogen (N1, blue) and the methyl carbon $(\mathrm{C} 2$, orange) of acetonitrile around TEMPO ${ }^{\bullet}$ (left) and TEMPO ${ }^{+}$ (right). Bottom panel: representative snapshots of the acetonitrile preferential orientation in the first solvation shell of $\mathrm{TEMPO}^{\bullet}$ (left) and $\mathrm{TEMPO}^{+}$(right). We use an isovalue of $28 \mathrm{~nm}^{-3}$ for $\mathrm{C} 2$ and $45 \mathrm{~nm}^{-3}$ for N1. Coordination numbers between the oxygen atom of TEMPO (O1) and either $\mathrm{C} 2$, N1 or center of mass (COM) of acetonitrile are respectively $2.9,1.1,3.1$ for TEMPO ${ }^{\bullet}$ and $2.2,2.9$ and 3.3 for $\mathrm{TEMPO}^{+}$. The cutoff values used to compute the coordination numbers are $4.4 \AA, 4.1 \AA$ and $4.8 \AA$ for O1-C2, O1-N1 and O1-COM, respectively.

2.9) and the missing peak in the O1-N1 RDF (with a coordination number of only 1.1). In contrast, the oxygen atom in $\mathrm{TEMPO}^{+}$is coordinated both to the methyl group and the nitrogen group of acetonitrile (the O1-C2 and O1-N1 coordination numbers are 2.2 and 2.9, respectively). The 3D-organization of $\mathrm{ACN}$ around TEMPO ${ }^{\bullet}$ and $\mathrm{TEMPO}^{+}$is furthermore visualized in Figure 7, depicting the spatial distribution functions (SDFs) of the methyl carbon (orange) and nitrogen (blue) of acetonitrile around the NO moiety. Only a very large orange isosurface is observed for TEMPO ${ }^{\bullet}$, while both orange and blue isosurfaces are observed for $\mathrm{TEMPO}^{+}$. In the latter case, this suggests that there is no preferential orientation of $\mathrm{ACN}$ in the first solvation shell of $\mathrm{TEMPO}^{+}$, with some molecules pointing toward the NO moiety with the methyl group, some with the nitrogen atom and some others with both groups. A representative snapshot is shown in Figure 7, bottom panel. This result suggests that there is only a difference in the orientation but not in the absolute number of acetonitrile molecules in the first solvation shell for the two redox states. In contrast, for AQ the first solvation shell of acetonitrile around the cabonyl oxygen becomes increasingly more crowded going from AQ to $\mathrm{AQ}^{2-}$.

\section{CONCLUSION}

In this work, a polarizable force-field was fitted to DFT calculations for AQ and TEMPO redox-active species in their various oxidation states as well as for acetonitrile. The structural organization of acetonitrile around AQ and TEMPO in both their oxidized and reduced states was sucessfully validated against previous DFT-MD simulations. We furthermore characterized their respective solvation shells in detail. An increase in the number of acetonitrile molecules around the AQ-oxygen atoms is found when going from AQ to $\mathrm{AQ}^{2-}$, which is accompanied by a change in the acetonitrile orientation. On the contrary, no changes in the total coordination number of $\mathrm{ACN}$ around the NO moiety are observed for TEMPO ${ }^{\bullet}$ and TEMPO $^{+}$, with only a difference in the preferential orientation.

The development of such a polarizable force field represents the first step toward the use of classical molecular dynamics simulations for the characterization of AQ and TEMPO redox-active molecules in many fields, for example in the case of biredox ionic liquids in supercapacitors. Calculating quantities such as the diffusion coefficients of the species adsorbed inside electrified nanopores will be possible and necessary to assess the power performance of the corresponding supercapacitors. Predicting the behavior of these molecules will also benefit other fields, since TEMPO is widely used in catalysis applications ${ }^{59}$ and $\mathrm{AQ}$ derivatives may be employed in redox-flow batteries in the future ${ }^{60,61}$.

\section{SUPPLEMENTARY MATERIAL}

See the supplementary material for Fumi-Tosi combinations rules, details of DFT calculations, force field parameters, intermolecular RDFs for acetonitrile, $\mathrm{AQ}$, $\mathrm{AQ}^{\bullet-}, \mathrm{AQ}^{2-}$, TEMPO ${ }^{\bullet}$ and $\mathrm{TEMPO}^{+}$, acetonitrile properties such as heat of vaporization, dielectric constant, diffusion coefficient and viscosity.

\section{ACKNOWLEDGMENTS}

This project has received funding from the European Research Council under the European Union's Horizon 2020 research and innovation programme (grant agreements No. 771294). This work was supported by the French National Research Agency (Labex STORE-EX, Grant ANR-10-LABX-0076). The authors acknowledge HPC resources granted by GENCI (resources of IDRIS, Grant No. A0100910463) and by the HPCaVe Centre at Sorbonne Université.

\footnotetext{
${ }^{1}$ E. M. Malik and C. E. Müller, "Anthraquinones as pharmacological tools and drugs," Med. Res. Rev. 36, 705-748 (2016).
} 
${ }^{2}$ P. Figiel, M. Leskelä, and T. Repo, "Tempo-copper(ii) diiminecatalysed oxidation of benzylic alcohols in aqueous media," Adv. Synth. Catal. 349, 1173-1179 (2007).

${ }^{3}$ M. Hayashi and Y. Shibuya, M.and Iwabuchi, "Oxidation of alcohols to carbonyl compounds with diisopropyl azodicarboxylate catalyzed by nitroxyl radicals," J. Org. Chem. 77, 30053009 (2012).

${ }^{4}$ M. R. Gerhardt, L. Tong, R. Gómez-Bombarelli, Q. Chen, M. P. Marshak, C. J. Galvin, A. Aspuru-Guzik, R. G. Gordon, and M. J. Aziz, "Anthraquinone derivatives in aqueous flow batteries," Adv. Energy Mater. 7, 1601488 (2017).

${ }^{5}$ X. Wei, W. Xu, M. Vijayakumar, L. Cosimbescu, T. Liu, V. Sprenkle, and W. Wang, "Tempo-based catholyte for highenergy density nonaqueous redox flow batteries," Adv. Mater. 26, 7649-7653 (2014).

${ }^{6} \mathrm{~J}$. Bitenc, N. Lindahl, A. Vizintin, M. E. Abdelhamid, R. Dominko, and P. Johansson, "Concept and electrochemical mechanism of an al metal anode - organic cathode battery," Energy Storage Mater. 24, 379-383 (2020).

${ }^{7}$ L. Yaoyao, C. Runyuan, S. Jiayue, L. Long, Z. Teng, and H. X., "Recent advances in coupling carbon-based electrode-redox electrolyte system," Mater. Res. Bull. 139, 111249 (2021).

${ }^{8}$ E. Mourad, L. Coustan, S. A. Freunberger, A. Mehdi, A. Vioux, F. Favier, and O. Fontaine, "Biredox ionic liquids: electrochemical investigation and impact of ion size on electron transfer," Electrochim. Acta 206, 513-523 (2016).

${ }^{9}$ E. Mourad, L. Coustan, P. Lannelongue, D. Zigah, A. Mehdi, A. Vioux, S. A. Freunberger, F. Favier, and O. Fontaine, "Biredox ionic liquids with solid-like redox density in the liquid state for high-energy supercapacitors," Nat. Mater. 16, 446-453 (2017).

${ }^{10}$ C. Bodin, E. Mourad, D. Zigah, S. Le Vot, S. A. Freunberger, F. Favier, and O. Fontaine, "Biredox ionic liquids: new opportunities toward high performance supercapacitors," Faraday Discuss. 206, 393-404 (2018).

${ }^{11}$ K. G. Reeves, A. Serva, G. Jeanmairet, and M. Salanne, "A first-principles investigation of the structural and electrochemical properties of biredox ionic species in acetonitrile," Phys. Chem. Chem. Phys. 22, 10561-10568 (2020).

${ }^{12}$ E. Stendardo, A. Pedone, P. Cimino, M. C. Menziani, O. Crescenzia, and V. Barone, "Extension of the amber forcefield for the study of large nitroxides in condensed phases: an ab initio parameterization," Phys. Chem. Chem. Phys. 12, 1169711709 (2010).

${ }^{13}$ M. Salanne, L. J. A. Siqueira, A. P. Seitsonen, P. A. Madden, and B. Kirchner, "From molten salts to room temperature ionic liquids: Simulation studies on chloroaluminate systems," Faraday Discuss. 154, 171-188 (2012).

${ }^{14}$ R. J. Heaton, R. Brookes, P. A. Madden, M. Salanne, C. a. Simon, and P. Turq, "A first-principles description of liquid bef2 and its mixtures with lif: 1. potential development and pure bef2," J. Phys. Chem. B 110, 11454-11460 (2006).

${ }^{15}$ M. P. Tosi and F. G. Fumi, "Ionic sizes and born repulsive parameters in the nacl-type alkali halides - ii: The generalized huggins-mayer form," J. Phys. Chem. Solids 25, 45-52 (1964).

${ }^{16}$ F. G. Fumi and M. P. Tosi, "Ionic sizes and born repulsive parameters in the nacl-type alkali halides - i: The huggins-mayer and pauling form," J. Phys. Chem. Solids 25, 31-43 (1964).

${ }^{17}$ K. T. Tang and J. P. Toennies, "An improved simple model for the van der waals potential based on universal damping functions for the dispersion coefficients," J. Chem. Phys. 80, 3726-3741 (1984).

${ }^{18}$ A. Aguado, L. Bernasconi, and M. Wilson, "Interionic potentials from ab initio molecular dynamics: The alkaline earth oxides cao, sro, and bao," J. Chem. Phys. 118 (2003).

${ }^{19}$ S. Jahn, P. A. Madden, and M. Wilson, Phys. Rev. B 69, 020106 (2004).

${ }^{20}$ A. Coretti, S. Bonella, and G. Ciccotti, "Communication: Constrained molecular dynamics for polarizable models," J. Chem. Phys. 149, 191102 (2018).
${ }^{21}$ E. Heid, P. A. Hunt, and C. Schröder, "Evaluating excited state atomic polarizabilities of chromophores," Phys. Chem. Chem. Phys. 20, 8554-8563 (2018).

${ }^{22}$ E. Heid, A. Szabadi, and C. Schröder, "Quantum mechanical determination of atomic polarizabilities of ionic liquids," Phys. Chem. Chem. Phys. 20, 10992-10996 (2018).

${ }^{23}$ M. J. Frisch, G. W. Trucks, H. B. Schlegel, G. E. Scuseria, M. A. Robb, J. R. Cheeseman, G. Scalmani, V. Barone, B. Mennucci, G. A. Petersson, H. Nakatsuji, M. Caricato, X. Li, H. P. Hratchian, A. F. Izmaylov, J. Bloino, G. Zheng, J. L. Sonnenberg, M. Hada, M. Ehara, K. Toyota, R. Fukuda, J. Hasegawa, M. Ishida, T. Nakajima, Y. Honda, O. Kitao, H. Nakai, T. Vreven, J. A. Montgomery, Jr., J. E. Peralta, F. Ogliaro, M. Bearpark, J. J. Heyd, E. Brothers, K. N. Kudin, V. N. Staroverov, R. Kobayashi, J. Normand, K. Raghavachari, A. Rendell, J. C. Burant, S. S. Iyengar, J. Tomasi, M. Cossi, N. Rega, J. M. Millam, M. Klene, J. E. Knox, J. B. Cross, V. Bakken, C. Adamo, J. Jaramillo, R. Gomperts, R. E. Stratmann, O. Yazyev, A. J. Austin, R. Cammi, C. Pomelli, J. W. Ochterski, R. L. Martin, K. Morokuma, V. G. Zakrzewski, G. A. Voth, P. Salvador, J. J. Dannenberg, S. Dapprich, A. D. Daniels, Farkas, J. B. Foresman, J. V. Ortiz, J. Cioslowski, and D. J. Fox, "Gaussian 09 revision e.01," Gaussian Inc. Wallingford CT 2009.

${ }^{24}$ A. J. Stone, "Distributed multipole analysis : stability of large basis sets," J. Chem. Theory Comput. 1, 1128-1132 (2005).

${ }^{25}$ A. J. Misquitta and A. J. Stone, "Distributed polarizabilities obtained using a constrained density-fitting algorithm," J. Chem. Phys. 124, 024111-1132 (2006).

${ }^{26}$ J. A. Lemkul, J. Huang, B. Roux, and A. D. MacKerell, "An empirical polarizable force field based on the classical drude oscillator model: Development history and recent applications," Chem. Rev. 116, 4983-5013 (2016).

${ }^{27}$ S. Grimme, J. Antony, S. Ehrlich, and H. Krieg, "A consistent and accurate ab initio parametrization of density functional dispersion correction (DF T-D) for the 94 elements H-Pu," J. Chem. Phys. 132, 154104 (2010).

${ }^{28}$ A. Marin-Laflèche, M. Haefele, L. Scalfi, A. Coretti, T. Dufils, G. Jeanmairet, S. K. Reed, A. Serva, R. Berthin, C. Bacon, S. Bonella, B. Rotenberg, P. A. Madden, and M. Salanne, "Metalwalls: A classical molecular dynamics software dedicated to the simulation of electrochemical systems," J. Open Source Softw. 5, 2373 (2020).

${ }^{29}$ L. Martínez, R. Andrade, E. G. Birgin, and J. M. Martínez, "Packmol: a package for building initial configurations for molecular dynamics simulations," J. Comput. Chem. 30, 2157-2164 (2009).

${ }^{30} \mathrm{~S}$. Nosé, "A unified formulation of the constant temperature molecular dynamics methods," J. Chem. Phys. 81, 511-519 (1984).

${ }^{31}$ D. J. Evans and B. L. Holian, "The nose-hoover thermostat," J. Chem. Phys. 83, 4069-4074 (1985).

${ }^{32}$ B. M. and B. Kirchner, "Travis - a free analyzer and visualizer for monte carlo and molecular dynamics trajectories," J. Chem. Inf. Model. 51, 2007-2023 (2011).

${ }^{33}$ X. Grabuleda, C. Jaime, and P. A. Kollman, "Molecular dynamics simulation studies of liquid acetonitrile: New six-site model," J. Comput. Chem. 21, 901-908 (2000).

${ }^{34}$ A. Nikitin and A. Lyubartsev, "New six-site acetonitrile model for simulations of liquid acetonitrile and its aqueous mixtures," J. Comput. Chem. 28, 2020-2026 (2007).

${ }^{35}$ H. J. Böhm, I. R. McDonald, and P. A. Madden, "An effective pair potential for liquid acetonitrile," Mol. Phys. 49, 347-360 (1983).

${ }^{36}$ J. M. Jorgensen, W. L.; Briggs, "Monte carlo simulations of liquid acetonitrile with a three-site model," Mol. Phys. 63, 547558 (1988).

${ }^{37}$ E. Guardia, R. Pinzón, J. Casulleras, M. Orozco, and F. Luque, "Comparison of different three-site interaction potentials for liquid acetonitrile," Mol. Simulat. 26, 287-306 (2001). 
${ }^{38}$ C. D. Wick, J. M. Stubbs, N. Rai, and J. I. Siepmann, "Transferable potentials for phase equilibria. 7. primary, secondary, and tertiary amines, nitroalkanes and nitrobenzene, nitriles, amides, pyridine, and pyrimidine," J. Phys. Chem. B 109, 18974-18982 (2005)

${ }^{39} \mathrm{~S}$. Pothoczki and L. Pusztai, "Intermolecular orientations in liquid acetonitrile: new insights based on diffraction measurements and all-atom simulations," J. Mol. Liq. 225, 160-166 (2007).

${ }^{40}$ T. Takamuku, M. Tabata, A. Yamaguchi, J. Nishimoto, M. Kumamoto, H. Wakita, and T. Yamaguchi, "Liquid structure of acetonitrile - water mixtures by x-ray diffraction and infrared spectroscopy," J. Phys. Chem. B 102, 8880-8888 (1998).

${ }^{41}$ E. Humphreys, P. Allan, R. Welbourn, T. Youngs, A. Soper, C. Grey, and S. Clarke, "A neutron diffraction study of the electrochemical double layer capacitor electrolyte tetrapropylammonium bromide in acetonitrile," J. Phys. Chem. B 119, 15320-15333 (2015).

${ }^{42} \mathrm{~J}$. William E. Acree, Infrared Spectra in NIST Chemistry WebBook, NIST Standard Reference Database Number 69 (http://webbook.nist.gov).

${ }^{43}$ J. Caillol, D. Levesque, and J. Weis, "Electrical properties of polarizable ionic solutions. i.theoretical aspects." J. Chem. Phys. 91, 5544-5554 (1989).

${ }^{44}$ J. Caillol, D. Levesque, and J. Weis, "Electrical properties of polarizable ionic solutions. ii.computer simulation results." J. Chem. Phys. 91, 5555-5566 (1989).

${ }^{45}$ V. A. Koverga, O. M. Korsun, O. N. Kalugin, B. A. Marekha, and A. Idrissi, "A new potential model for acetonitrile: Insight into the local structure organization," J. Mol. Liq. 233, 251-261 (2017).

${ }^{46} \mathrm{~J}$. G. McDaniel, "Polarization effects in binary $\left[\mathrm{bmim}^{+}\right]\left[\mathrm{bf}^{-}\right] / 1,2$-dichloroethane, acetone, acetonitrile, and water electrolytes," J. Phys. Chem. B. 122, 4345-4355 (2018).

${ }^{47} \mathrm{P}$. Howard and I. Wadsö, "Enthalpies of vaporization of organic compounds. iv. alkyl nitriles." Acta Chem. Scand. 24, 145-149 (1970).

${ }^{48}$ D. S. Venables and C. A. Schmuttenmaer, "Far-infrared spectra and associated dynamics in acetonitrile-water mixtures measured with femtosecond thz pulse spectroscopy," J. Chem. Phys. 108, 4935-4944 (1998).

${ }^{49}$ N. van Meurs and G. Somsen, "Excess and apparent molar volumes of mixtures of water and acetonitrile between 0 and 25c,"
J. Solution Chem. 22, 427-436 (1993).

${ }^{50}$ J. Coetzee and M. Martin, "Acetonitrile," in Recommended Methods for Purification of Solvents and Tests for Impurities, edited by J. COETZEE (Pergamon, 1982) pp. $10-15$.

${ }^{51}$ H. Kovacs, J. Kowalewski, A. Maliniak, and P. Stilbs, "Multinuclear relaxation and nmr self-diffusion study of the molecular dynamics in acetonitrile-chloroform liquid mixtures," J. Phys. Chem. 93, 962-969 (1989).

${ }^{52}$ H. Shekaari, M. Zafarani-Moattar, and M. S.N., "Density, viscosity, speed of sound, and refractive index of a ternary solution of aspirin, 1-butyl-3-methylimidazolium bromide, and acetonitrile at different temperatures $\mathrm{t}=(288.15$ to 318.15$) \mathrm{k}, " \mathrm{~J}$. Chem. Eng. Data 60, 1572-1583 (2015).

${ }^{53}$ X.-W. An and M. Månsson, "Enthalpies of combustion and formation of acetonitrile," J. Chem. Thermodyn. 15, 287-293 (1983).

${ }^{54} \mathrm{M}$. Grande, H. Bianchi, and C. Marschoff, "On the density of pure acetonitrile," An. Asoc. Quim. Argent. 92, 109-114 (2004).

${ }^{55}$ M. Holz, X. Mao, D. Seiferling, and A. Sacco, "Experimental study of dynamic isotope effects in molecular liquids: Detection of translation-rotation coupling," J. Chem. Phys. 93, 669-679 (1996).

${ }^{56}$ J. Baldt and H. Hall, "Thermochemistry of strained-ring bridgehead nitriles and esters," J. Am. Chem. Soc. 93, 140-145 (1971).

${ }^{57}$ D. Grant-Taylor and D. Macdonald, "Thermal pressure and energy-volume coefficients for the acetonitrile + water system," Can. J. Chem. 54, 2813-2819 (1976).

${ }^{58}$ M. Liang, X. Zhang, A. Kaintz, N. Ernsting, and M. Maroncelli, "Solvation dynamics in a prototypical ionic liquid + dipolar aprotic liquid mixture: 1-butyl-3-methylimidazolium tetrafluoroborate + acetonitrile," J. Phys. Chem. B 118, 13401352 (2014)

${ }^{59}$ H. A. Beejapur, Q. Zhang, K. Hu, L. Zhu, J. Wang, and Z. Ye, "Tempo in chemical transformations: From homogeneous to heterogeneous," ACS Catal. 9, 2777-2830 (2019).

${ }^{60}$ S. Er, C. Suh, M. P. Marshak, and A. Aspuru-Guzik, "Computational design of molecules for an all-quinone redox flow batterycomputational design of molecules for an all-quinone redox flow battery," Chem. Sci. 6, 885-893 (2015).

${ }^{61}$ M. T. Huynh, C. W. Anson, A. C. Cavell, S. S. Stahl, and $\mathrm{S}$. Hammes-Schiffer, "Quinone $1 \mathrm{e}-$ and $2 \mathrm{e}-/ 2 \mathrm{~h}+$ reduction potentials: Identification and analysis of deviations from systematic scaling relationships," J. Am. Chem. Soc. 138, 1590315910 (2016). 